\title{
A major QTL corresponding to the $R k$ locus for resistance to root-knot nematodes in cowpea (Vigna unguiculata $\mathbf{L}$. Walp.)
}

\author{
Bao-Lam Huynh ${ }^{1}$ - William C. Matthews ${ }^{1} \cdot$ Jeffrey D. Ehlers $^{2} \cdot$ Mitchell R. Lucas $^{3}$ • \\ Jansen R. P. Santos ${ }^{1} \cdot$ Arsenio Ndeve $^{1} \cdot$ Timothy J. Close $^{3} \cdot$ Philip A. Roberts $^{1}$
}

Received: 25 June 2015 / Accepted: 21 September 2015 / Published online: 8 October 2015

(C) The Author(s) 2015. This article is published with open access at Springerlink.com

\begin{abstract}
Key message Genome resolution of a major QTL associated with the $R k$ locus in cowpea for resistance to root-knot nematodes has significance for plant breeding programs and $\mathbf{R}$ gene characterization.

Abstract Cowpea (Vigna unguiculata L. Walp.) is a susceptible host of root-knot nematodes (Meloidogyne spp.) (RKN), major plant-parasitic pests in global agriculture. To date, breeding for host resistance in cowpea has relied on phenotypic selection which requires time-consuming and expensive controlled infection assays. To facilitate markerbased selection, we aimed to identify and map quantitative trait loci (QTL) conferring the resistance trait. One recombinant inbred line (RIL) and two F2:3 populations, each derived from a cross between a susceptible and a resistant parent, were genotyped with genome-wide single nucleotide polymorphism (SNP) markers. The populations were screened in the field for root-galling symptoms and/or
\end{abstract}

Communicated by B. Diers.

Electronic supplementary material The online version of this article (doi:10.1007/s00122-015-2611-0) contains supplementary material, which is available to authorized users.

Bao-Lam Huynh

baolam.huynh@ucr.edu

Philip A. Roberts

philip.roberts@ucr.edu

1 Department of Nematology, University of California, Riverside, CA 92521, USA

2 Present Address: Bill and Melinda Gates Foundation, Seattle, WA 98102, USA

3 Department of Botany and Plant Sciences, University of California, Riverside, CA 92521, USA under growth-chamber conditions for nematode reproduction levels using $M$. incognita and M. javanica biotypes. One major QTL was mapped consistently on linkage group VuLG11 of each population. By genotyping additional cowpea lines and near-isogenic lines derived from conventional backcrossing, we confirmed that the detected QTL co-localized with the genome region associated with the $R k$ locus for RKN resistance that has been used in conventional breeding for many decades. This chromosomal location defined with flanking markers will be a valuable target in marker-assisted breeding and for positional cloning of genes controlling RKN resistance.

\section{Introduction}

Root-knot nematodes (Meloidogyne spp.) (RKN) are among the most devastating soil-borne crop pests worldwide, causing annual losses amounting to billions of dollars in global agriculture (Abad et al. 2008). They parasitize plant root systems and thus directly affect the uptake of water and nutrients needed for normal plant growth and reproduction. Their infection of plant roots can also be components of disease complexes with other pathogens including vascular diseases such as Fusarium wilt and root rots (Roberts et al. 1995). Cowpea (Vigna unguiculata L. Walp.) is a common host of RKN; it is grown in the USA and on larger scales in semi-arid regions of Africa and other warm to hot regions where RKN is prevalent (Ehlers and Hall 1997; Olowe 2004; Sawadogo et al. 2009). Several RKN species are known to attack cowpea, among which $M$. incognita and $M$. javanica are most widespread (Fery and Dukes 1980; Roberts et al. 2005). Due to cost and safety limitations on the use of chemical nematicides, nematode management in cowpea includes alternative strategies 
involving crop rotations and host resistance (Roberts et al. 2005). Using nematode-resistant cowpea cultivars as a grain crop or as cover crops in cropping systems can promote yield and suppress nematode populations in the soil during crop rotation (Roberts et al. 2005).

Quantitative inheritance studies have indicated that RKN resistance in cowpea involves different additive genes (Ehlers et al. 2000b; Fery and Dukes 1980; Fery et al. 1994; Roberts et al. 1996). Of these, the $R k$ gene, designated by Fery and Dukes in 1980, is effective against different RKN isolates and thus has been deployed in modern cultivars via conventional breeding using phenotypic selection (Ehlers et al. 2002, 2009). Until now, there has not been a marker-based definition of the $R k$ locus to facilitate indirect selection of the resistance trait in breeding programs. Breeders have relied on field, greenhouse and controlled environment techniques for resistance phenotyping; however, these approaches are expensive, time-consuming and often hindered by inaccuracies due to variations in phenotyping assays. Field screening for resistance to nematodes involves the use of isolated plots that are managed to maintain high and uniform nematode population densities through systematic crop rotation protocols with susceptible crops in non-screening years. Plants grown in infested field plots are visually rated for extent of root-galling. Controlled inoculation screens using greenhouse-grown potted plants or in seedling growth pouches grown in controlled environment chambers have also been developed and are more accurate than field screens but are also more laborintensive (Ehlers et al. 2000b; Omwega et al. 1988; Roberts et al. 1996). While some phenotyping is necessary in marker-assisted selection breeding, development of markers for indirect selection of nematode resistance has many advantages, especially when high-throughput genotyping platforms are available, as they are now for cowpea (Huynh et al. 2015; Muchero et al. 2009). In this study we report on the identification of a major QTL in the cowpea genome which contains the $R k$-gene trait determinant, with defined flanking markers based on genic-SNPs. This paves the way for deployment of a more efficient breeding strategy using genetic markers.

\section{Materials and methods}

\section{Mapping populations}

Three cowpea segregating populations used in genetic mapping included (1) 87 recombinant inbred lines (RILs) from the cross CB27 $\times 24-125 B-1$, (2) 170 F2:3 families from the cross IT84S-2049 $\times$ UCR779 and (3) 132 F2:3 families from the cross IT93K-503-1 $\times$ UCR779. CB27 is a California Blackeye dry grain variety bred by University of California-Riverside (UCR) (Ehlers et al. 2000a) while IT84S-2049 and IT93K-503-1 are breeding lines developed by the International Institute of Tropical Agriculture (IITA, Nigeria), all of which are highly resistant to RKN. The breeding line 24-125B-1 was developed by the Institute de Recherche Agricole pour le Development (IRAD, Cameroon) and UCR 779 is a Botswana cowpea landrace donated by de Moy (Colorado State University) in 1987, both of which are highly susceptible to $M$. incognita and M. javanica.

\section{Nematode isolates}

Three root-knot nematode isolates were used for resistance screening. The M. incognita isolates 'Project 77' and 'Beltran' originally isolated from cotton and lima bean fields, respectively, in the San Joaquin Valley, California, were characterised previously to be avirulent on cowpeas with resistance conferred by the $R k$ gene (Roberts et al. 1995). The $M$. javanica isolate ' 811 ' is aggressive on cowpea plants with $R k$ resistance, rendering the resistance response partially effective, and was isolated from a cowpea field in southern California. All isolates have been maintained on greenhouse-grown susceptible tomato plants (Thomason and McKinney 1960).

\section{Resistance phenotyping: egg-mass production}

The RIL population CB27 $\times 24-125 \mathrm{~B}-1$ and parents were assayed for RKN resistance using a seedling growth-pouch inoculation system described in Ehlers et al. (2000b) and visualized in Atamian et al. (2012). In brief, cowpea seeds from each RIL were germinated in a petri dish. Each germinated seed was then transferred into one pouch. The pouches were watered daily with distilled water and kept in a controlled environment growth chamber with constant temperature $\left(27^{\circ} \mathrm{C}\right)$ and $16 \mathrm{~h}$ of light per day. When adequate root systems were developed (about 14 days), each pouch was inoculated with approximately $1500 \mathrm{sec}-$ ond-stage juveniles of $M$. incognita isolate project 77 . The nematode juvenile inoculum was prepared by hatching nematode eggs extracted from tomato roots. After inoculation, the plants were maintained in Hoagland's growth solution (Hoagland and Arnon 1950) for 30 days and then treated with egg-mass-selective erioglaucine dye (Sigma Chemical Co., St. Louis, MO, USA) overnight. Stained egg masses on plant roots were counted with the aid of a $10 \times$-illuminated magnifier. The experiment was arranged in a randomized complete block design with three replications, each containing one seedling per pouch per RIL. Mean number of egg masses per RIL was used to classify resistance levels. 


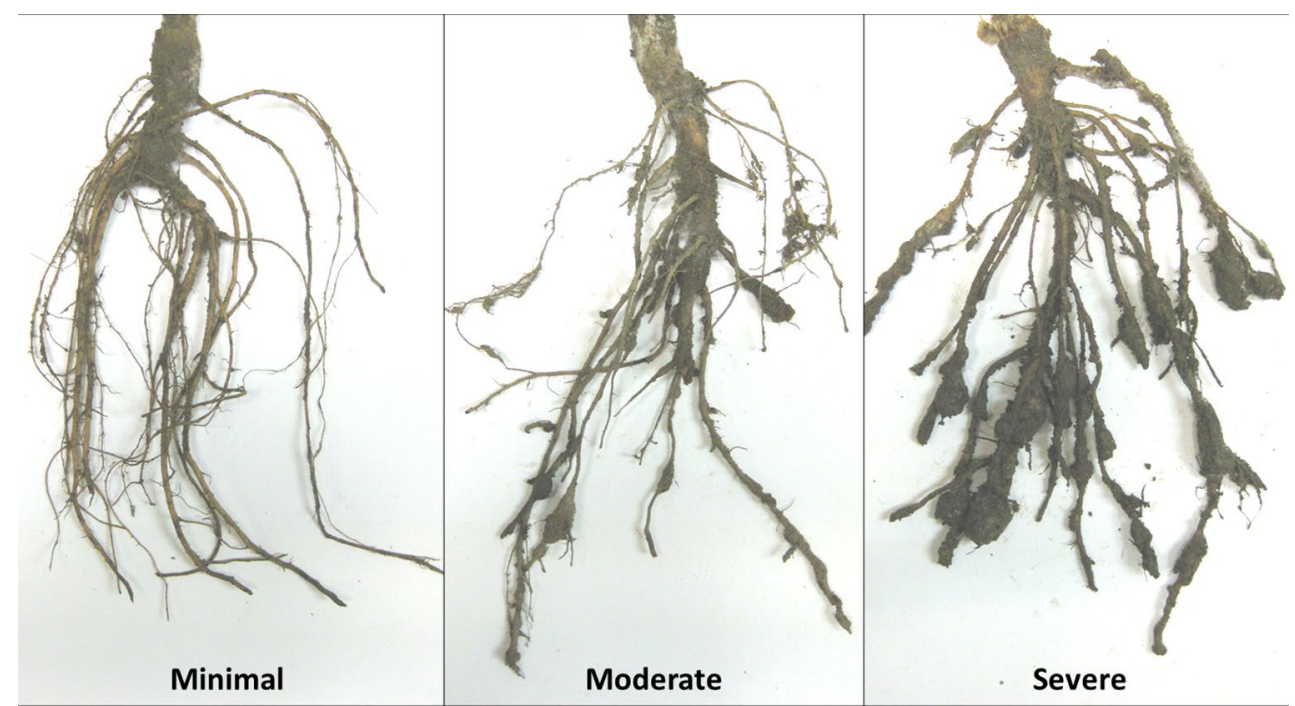

Fig. 1 Examples of root-galling symptoms induced by root-knot nematodes observed in the phenotype screens of mapping populations grown in the field tests

\section{Resistance phenotyping: root-galling symptoms}

The mapping populations plus parents were evaluated at the University of California South Coast Research and Extension Center (SCREC). In 2008, the CB27 × 24-125B-1 RIL population was tested with $M$. javanica isolate 811 . The IT84S-2049 $\times$ UCR779 and IT93K-503-1 × UCR779 F2:3 populations were tested with $M$. incognita isolate Beltran in 2010 and 2012, respectively. In 2012, the CB27 x 24-125B-1 RILs were screened in separate field blocks, one infested with $M$. incognita isolate Beltran and the other infested with $M$. javanica isolate 811 . The field sites contained root-knot nematode infestations which were established by injecting an inoculum of nematode eggs extracted from greenhouse-grown tomato plants into the root-zone of young susceptible tomato plants, followed by several years of growing susceptible tomatoes to provide high and uniform infestation levels. Nematode-induced galling symptoms on the tomato root systems were scored throughout the experimental block as a bio-assay before the cowpea experiment in each site, ensuring that the infestation levels were at least a score 7-8 using the root-gall rating chart of Bridge and Page (1980). Nematode species identification was verified by isozyme profiles and the N. Carolina host differential test of the original inocula and rechecked by these methods and with species-specific DNA primers by PCR prior to the cowpea experiments. Each RIL or F2:3 family was grown in one 1.5-m-long plot, with 20-25 plants per line or family. Uniformity and intensity of RKN infection in all experiments were confirmed on the basis of similar mean plot scores of susceptible parents (24-125B-1 and UCR779) that were grown next to the resistant parent in 5 replications (20-25 plants per replicate) throughout the experiment as positive controls for nematode infection. After 60-70 days, the plant tops were cut and each root system dug and scored for galling symptoms using a scale from 0 (no symptoms) to 9 (severe galling), modified from the rating chart of Bridge and Page (1980) (Fig. 1).

For the CB27 × 24-125B-1 RIL population, Analysis of Variance (ANOVA) was performed with the software GenStat version 11 (Payne et al. 2008). Factors in the ANOVA model were lines and block, with each field and year considered as a block. Broad-sense heritability was estimated based on the variance component attributable to variation among lines (VG) and residual variation (VE) $\left[h^{2}=\mathrm{VG} /\right.$ $(\mathrm{VG}+\mathrm{VE})]$. Simple linear correlation analysis was used to examine the phenotypic relationship between egg-mass numbers on pouch-grown root systems and root-galling symptoms measured in different field screenings. For F2:3 populations, mean score of galling symptoms per family was used to classify resistance levels.

\section{Linkage analyses and QTL mapping}

Genotypic data of 1536 genome-wide SNP markers (Muchero et al. 2009) for the CB27 × 24-125B-1 RIL population were obtained from Lucas et al. (2011). For two F2 populations (IT84S-2049 $\times$ UCR779 and IT93K-503-1 $\times$ UCR779), F2 individuals of each population were genotyped with the Kompetitive allele-specific polymerase chain reaction (KASP) assay (LGC Genomics Ltd., Hoddesdon, UK) (Semagn et al. 2014) using 168 and 155 SNP markers, respectively. These markers were polymorphic between the two parents of each corresponding population and spaced 
at least $2 \mathrm{cM}$ apart on the cowpea consensus genetic map (Lucas et al. 2011) [also accessible at HarvEST:Cowpea 1.33 (http://harvest.ucr.edu/)]. Linkage-map construction was performed with the software QTL IciMapping 4.0 (http://www.isbreeding.net) using the Kosambi function, RECORD ordering algorithm (Van Os et al. 2005) and alignment with the cowpea consensus genetic map.

The Inclusive Composite Interval Mapping (ICIM) method ( $\mathrm{Li}$ et al. 2007; Wang 2009) was also performed with the software QTL IciMapping 4.0. ICIM involved three consecutive steps: (1) significant markers associated with phenotypes were selected through single marker analysis; (2) the selected markers, except for two markers flanking the current mapping interval, were used to adjust phenotypic values; and (3) the adjusted phenotypic values were used in composite interval mapping (Yang et al. 2007) for QTL identification.

\section{Genotyping of near-isogenic lines (NILs)}

Three NILs that differ in the presence or absence of the $R k$-resistance were developed using conventional backcrossing. In brief, the recurrent parent CB46 (homozygous resistant, RkRk) (Helms et al. 1991) was crossed with a highly susceptible cowpea landrace 'Chinese Red' (homozygous susceptible, rkrk), and then the F1 was backcrossed to the recurrent parent CB46 to generate BC1F1. Blind crosses were then made between $\mathrm{BC} 1 \mathrm{~F} 1$ plants with CB46, and selfed seeds (BC1F2) of each BC1F1 plant were screened with $M$. incognita isolate Project 77 in growth pouches for variation in RKN resistance based on eggmass production, in comparison with the parents CB46 and Chinese Red. Subsequently, crosses made from heterozygous resistant $\mathrm{BCF} 1$ plants (Rkrk), whose $\mathrm{BC} 1 \mathrm{~F} 2$ segregated for resistance, were selected for the next backcross cycle. Backcrossing was repeated for six cycles followed by single seed descent and phenotyping at the F6 to select for homozygous susceptible lines. Two highly susceptible lines (Null 1 and Null 2) were genotyped with 1536 SNPs using the Illumina GoldenGate assay. Marker profiles of these lines and the original parent plants were aligned with the cowpea consensus genetic maps (Lucas et al. 2011) for identification of regions that coincided with QTLs detected in mapping populations.

\section{Results}

\section{Resistance variation}

The production of egg-masses (EM) varied widely among the CB27 $\times 24-125 B-1$ RILs grown in pouches tested with M. incognita, ranging from 0 to 208 mean EM per root system (Fig. 2a). The segregation appeared to follow a bimodal distribution, with the resistant parent CB27 showing a lower mean EM number than the susceptible parent 24-125B-1. In field experiments, root-galling (RG) scores also varied widely, ranging from 0 to 9 (Fig. 2b, c, d). The distributions were skewed toward the lower phenotypic value in the $M$. incognita tests, whereas in $M$. javanica tests the distributions were skewed toward the higher phenotypic value. The RG data for the RIL population were quite repeatable across years and nematode fields, with broadsense heritability estimated at 0.8 . There were also significant correlations $(r>0.45, P<0.001)$ between EM and RG data, with resistant $\mathrm{CB} 27$ showing lower phenotypic values than susceptible 24-125B-1 in all experiments.

Large variation in RG symptoms was also observed among F2:3 families of the crosses IT84S-2049 $\times$ UCR779 (Fig. 2e) and IT93K-503-1 × UCR779 (Fig. 2f) when grown in the field tested with $M$. incognita. Resistant parents IT84S-2049 and IT93K-503-1 showed lower RG scores than the susceptible parent UCR779. The distribution appeared skewed toward lower phenotypic values in both mapping populations.

\section{QTL identification}

A QTL with major effect on EM production and RG symptoms was consistently mapped in the same region on linkage group VuLG11 of the CB27 $\times 24-125 B-1$ RIL population using data from four separate $\mathrm{RKN}$ phenotyping assays (Fig. 3). The QTL effect was highest in the EM assay with M. incognita, explaining $73 \%$ of total phenotypic variance (PVE), followed by field screens with M. incognita in 2012 $(\mathrm{PVE}=71 \%)$ and $M$. javanica in $2008(\mathrm{PVE}=59 \%)$ and $2012(\mathrm{PVE}=52 \%)$ (Table 1). The favourable (low EM numbers and RG scores) allele was contributed from the resistant parent CB27.

A major QTL associated with RG symptoms was also mapped on linkage group VuLG11 of each F2:3 population when assayed with $M$. incognita in the field in 2010 (Fig. 4). The QTL effect was higher in the IT84S-2049 $\times$ UCR779 population $(\mathrm{PVE}=83 \%)$ than in the IT93K503-1 $\times$ UCR779 population $(\mathrm{PVE}=65 \%)($ Table 1 ; Fig. 4); in both F2:3 populations, the QTL showed partial dominance, and lower RG scores were conferred by the presence of the IT84S-2049 and IT93K-503-1 alleles. SNP markers nearest to this QTL (1_0757 and 1_0414) also flanked the major QTL detected in the CB27 × 24-125B-1 RIL population (Fig. 3).

\section{The $R k$ location}

Genotyping of the resistant CB46 and two susceptible NILs (Null 1 and Null 2) revealed that the NILs were 

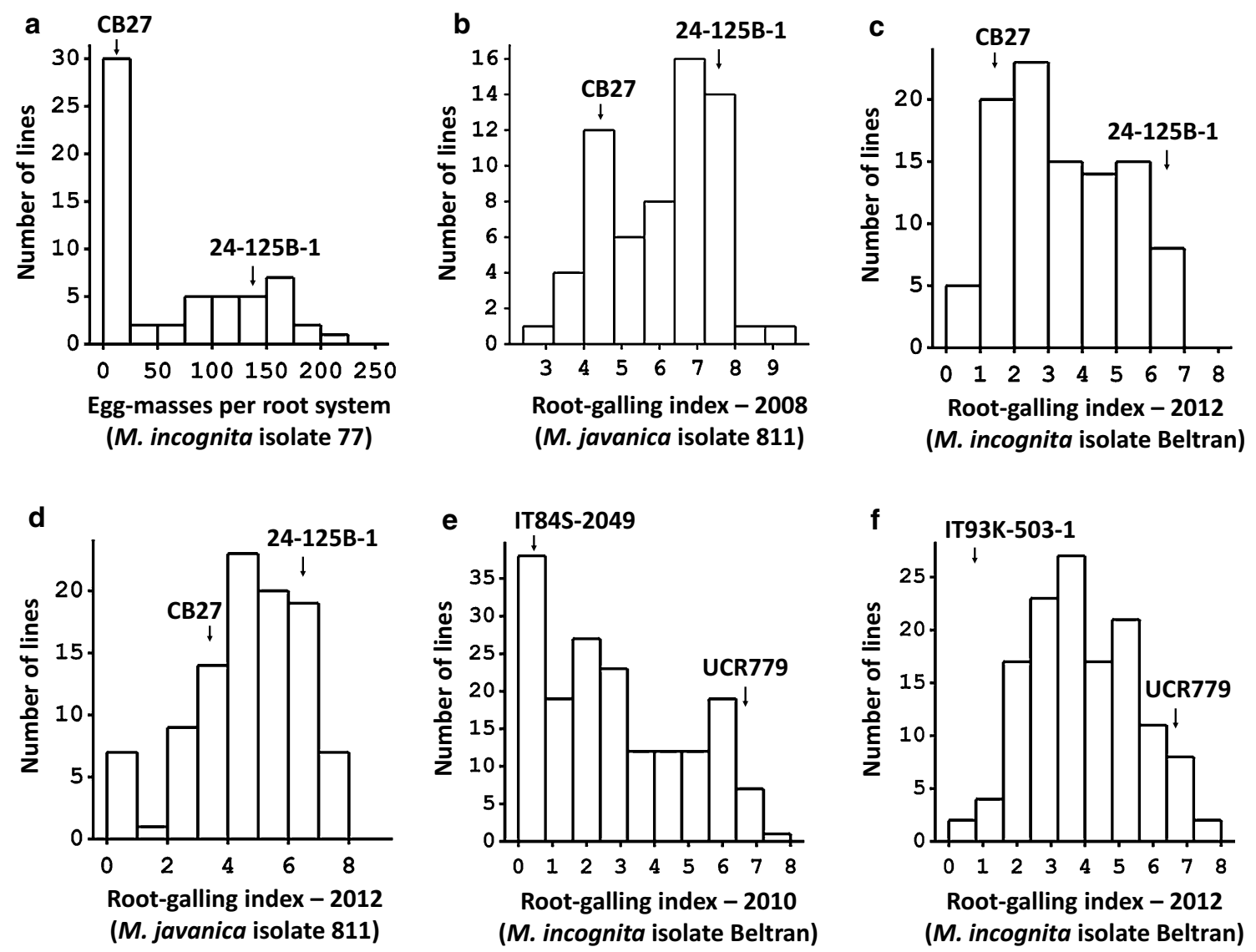

Fig. 2 Variation in response to root-knot nematode infection within the cowpea populations $(\mathbf{a}-\mathbf{d})$ CB27 $\times$ 24-125B-1 RIL, (e) IT84S$2049 \times$ UCR779 F2:3, and (f) IT93K-503-1 × UCR779 F2:3 phenotyped in multiple tests with different $\mathrm{RKN}$ isolates (in parentheses).
Mean phenotypic values for the parents in each test are indicated by labelled arrows. The number of RKN egg-masses per root system was measured in inoculated growth pouches. Root-galling was indexed in infested field plots
Fig. 3 Chromosomal locations on cowpea linkage group VuLG11 of the CB27 $\times$ 24-125B-1 RIL population associated with the variation in root-knot nematode egg-mass production (EM) and rootgalling symptoms (RG) in four experiments

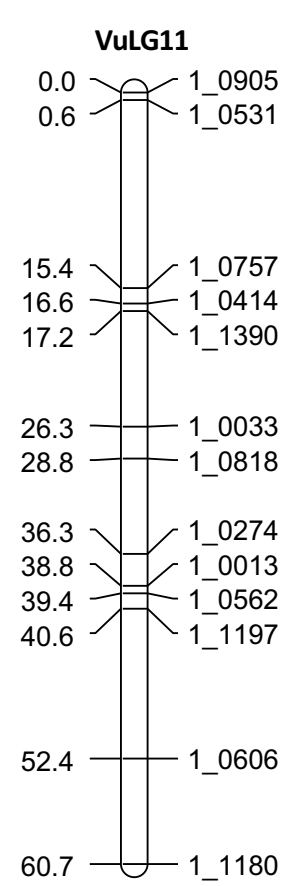


Table 1 Chromosome locations associated with resistance to different root-knot nematode species and isolates in cowpea RIL and F2:3 populations phenotyped in inoculated seedling growth pouches and infested field plots

\begin{tabular}{|c|c|c|c|c|c|c|c|c|c|}
\hline $\begin{array}{l}\text { Mapping population } \\
(\text { female } \times \text { male) }\end{array}$ & $\begin{array}{l}\text { Resistance trait (envi- } \\
\text { ronments, RKN isolates) }\end{array}$ & $\begin{array}{l}\text { Linkage } \\
\text { group }\end{array}$ & Position (cM) & $\begin{array}{l}\text { Flanking } \\
\text { markers }\end{array}$ & LOD & $\operatorname{PVE}(\%)^{\mathrm{a}}$ & $\operatorname{Add}^{\mathrm{b}}$ & $\operatorname{Dom}^{c}$ & $\mathrm{D} / \mathrm{A}^{\mathrm{d}}$ \\
\hline \multirow[t]{4}{*}{$\begin{array}{r}\text { RIL (CB27 × } \\
24-125 B-1)\end{array}$} & $\begin{array}{l}\text { Egg masses (growth } \\
\text { pouches, } M \text {. incognita } \\
77 \text { ) }\end{array}$ & VuLG11 & 16 & 1_0757-1_0414 & 27.0 & 72.9 & -57.6 & NA & NA \\
\hline & $\begin{array}{l}\text { Gall score (field } 2012, \\
\text { M. incognita Beltran) }\end{array}$ & VuLG11 & 14 & 1_0531-1_0757 & 16.0 & 70.9 & -1.5 & NA & NA \\
\hline & $\begin{array}{l}\text { Gall score (field 2008, } \\
\text { M. javanica } 811 \text { ) }\end{array}$ & VuLG11 & 15 & 1_0531-1_0757 & 10.2 & 59.2 & -1.0 & NA & NA \\
\hline & $\begin{array}{l}\text { Gall score (field 2012, } \\
\text { M. javanica } 811 \text { ) }\end{array}$ & VuLG11 & 13 & 1_0531-1_0757 & 8.2 & 52.4 & -1.4 & NA & NA \\
\hline $\begin{array}{l}\text { F2:3 (IT84S-2049 × } \\
\text { UCR779) }\end{array}$ & $\begin{array}{l}\text { Gall score (field } 2010, \\
\text { M. incognita Beltran) }\end{array}$ & VuLG11 & 19 & 1_0905-1_0414 & 60.8 & 83.1 & -2.7 & -0.7 & 0.26 \\
\hline $\begin{array}{l}\text { F2:3 (IT93 K-503-1 } \times \\
\text { UCR779) }\end{array}$ & $\begin{array}{l}\text { Gall score (field 2010, } \\
\text { M. incognita Beltran) }\end{array}$ & VuLG11 & 14 & 1_0886-1_0757 & 29.3 & 64.5 & -1.8 & -0.4 & 0.22 \\
\hline
\end{tabular}

${ }^{\text {a }}$ Percentage of variance explained

b Additive effects: negative values indicate that alleles from the susceptible male parents contributed to higher phenotypic values

${ }^{c}$ Dominance effects: negative values indicate that the mean of lines heterozygous for the QTL is lower than the phenotypic value half way between the mean of lines homozygous for one allele and that of lines homozygous for the alternative allele (NA not applicable in the RIL population)

${ }^{d}$ The dominance/additive ratios showing gene action modes as partial dominance $(\mathrm{D} / \mathrm{A}=0.21-0.80)$

almost completely homozygous for the CB46 background except for a common region of about $6 \mathrm{cM}$ on VuLG11 of the cowpea consensus genetic map that was homozygous for the susceptible donor (Chinese Red) alleles (Fig. 5 and Supplementary File). This region contains SNP markers (1_0531, 1_0894 and 1_0757) that also flank the major QTL mapped on VuLG11 in the three mapping populations (Figs. 3 and 4).

\section{Discussion}

The skewed or bi-modal phenotypic distributions observed for resistance traits, based on nematode reproduction and root-galling symptoms (Fig. 2), support the presence of a major QTL which was detected in each mapping population. Given their common proximity to marker 1_0414, the three QTLs mapped in separate populations (Figs. 3 and 4) could be confined to a single multi-allelic locus, hereafter designated $Q R k$-vul1.1. By genotyping of $R k$ and non- $R k$ NILs derived from conventional backcrossing, we confirmed that $Q R k$-vull.1 corresponds to the $R k$ locus, which was first designated by Fery and Dukes (1980) and has been used in conventional breeding of resistant cowpea for a long time (Ehlers et al. 2000a, 2009; Hall et al. 2003; Hall and Frate 1996). Based on cowpea consensus genetic maps (Lucas et al. 2011), QRk-vull.1 is not in the same locations of previously published QTLs for resistance to other biotic traits such as fungal diseases (Muchero et al. 2011; Pottorff et al. 2012, 2014), viruses (Ouédraogo et al. 2002), the parasitic weed Striga (Ouédraogo et al. 2012) and insects (Huynh et al. 2015; Lucas et al. 2012). QRk-vu11.1 could be deployed in breeding programs by SNP-genotyping to select directly for the $R k$ locus, minimizing the need for laborious controlled phenotype assays.

The two susceptible null- $R k$ lines were developed following the conventional scheme applied in $\mathrm{RKN}$ resistance breeding (Ehlers et al. 2002). It took more than 5 years from the original cross between the recurrent parent CB46 and the susceptible donor Chinese Red to develop BC6F6 lines for field evaluation; each backcross cycle required at least 5 months for blind crossing, collecting selfed-seeds and then phenotyping the selfed-seeds to decide which cross to advance. Genotyping of BC6F6 lines (Fig. 5, Supplementary File), however, revealed substantial linkage drag in some lines that extended many centimorgans beyond QTL-flanking markers. Since a cM may be hundreds of kilobases in length (Dohm et al. 2012; Zhang et al. 2012), with the $620 \mathrm{Mb}$ cowpea genome having an average genetic map length of $680 \mathrm{cM}$ (Lucas et al. 2011; Muchero et al. 2009), conventional backcrossing potentially introduced many 'unwanted' genes from resistance donors. With marker-assisted backcrossing, desirable recombinants with minimal linkage drag could be selected based on both flanking and adjacent markers around $Q R k$-vul1.1. Furthermore, combining this foreground selection approach with background selection for the recurrent parent genotype 
Fig. 4 Chromosomal locations on linkage group VuLG11 of the (a) IT84S-2049 $\times$ UCR779 and (b) IT93K-503-1 $\times$ UCR779 F2 populations associated with the variation in root-galling symptoms expressed in the F2:3 families grown in field plots infested with $M$. incognita isolate Beltran in 2010 and 2012, respectively a VuLG11

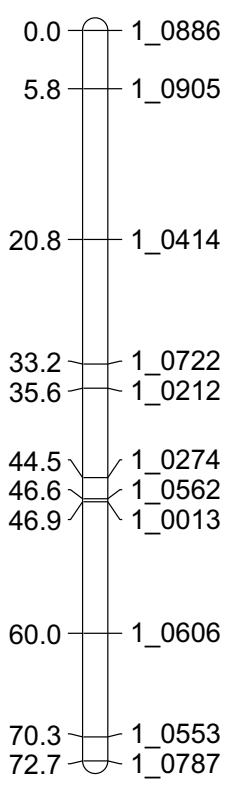

b VuLG11

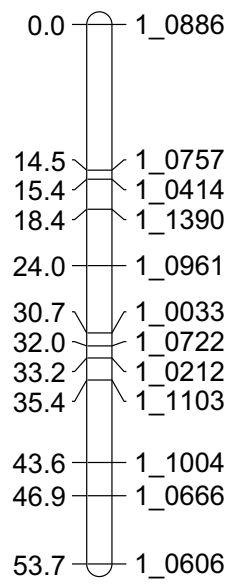

LOD

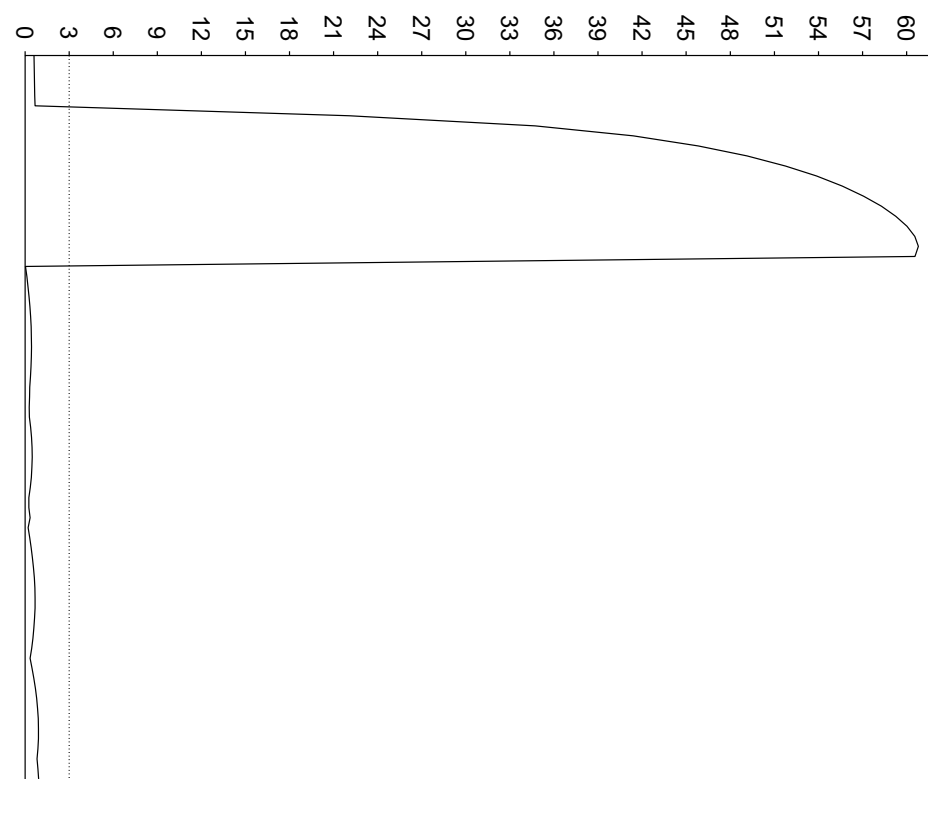

LOD

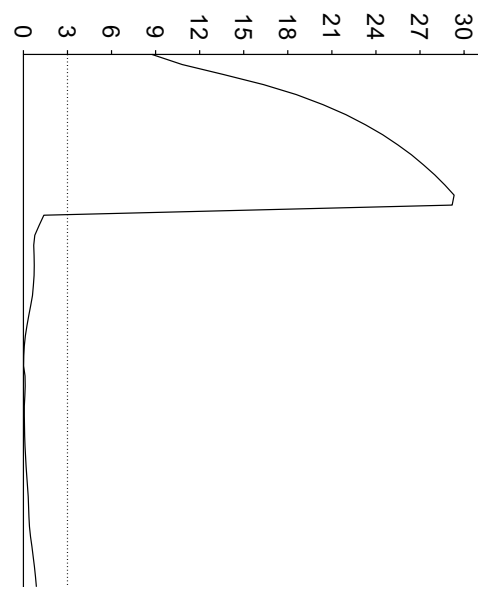

using available genome-wide markers could expedite the breeding process.

The nematode isolates used in this study represent two main RKN species ( $M$. incognita and $M$. javanica) that cause substantial damage to cowpea in the USA and most cowpea-growing regions worldwide (Petrillo et al. 2006). Based on genetic mapping, the QTL $Q R k$-vu11.1 exhibited a major effect on resistance against both species, albeit with a lower effect on $M$. javanica (Table 1). This is in contrast to soybean where QTLs conferring resistance to $M$. incognita and $M$. javanica were mapped on different chromosomes (Tamulonis et al. 1997; Xu et al. 2013), indicating a broad functionality of the $R k$ locus in the cowpea system. Variation in the effectiveness of the $R k$-based resistance to different isolates of these two species in cowpea has been documented (Petrillo et al. 2006; Petrillo and Roberts 2005). This variation reflects differences in virulence among $M$. incognita populations, with evidence of selection for virulence to $R k$ in fields routinely planted with cowpea cultivars carrying the $R k$ gene, while some $M$. javanica populations are more aggressive on $R k$ plants than avirulent $M$. incognita. Nevertheless, the broad utility of this resistance to most root-knot nematode populations in cowpea production areas is highly valuable for nematode management programs. Furthermore, our positioning of the $R k$ locus on the cowpea genetic map provides an important step in the comparative characterization of other RKN resistance genes in cowpea which hitherto have been identified by non-marker-based inheritance studies (Ehlers et al. 2000b, 2002; Roberts et al. 1996).

Previous studies showed that $R k$-resistance did not suppress root penetration by infective juveniles of $M$. 


\begin{tabular}{|c|c|c|c|c|c|}
\hline \multicolumn{2}{|c|}{ VuLG11 } & CB46 & Chinese Red & Null 1 & Null 2 \\
\hline & |1_0886 & AA & GG & GG & AA \\
\hline 3.2 & 1_-0702 & AA & $\mathrm{CC}$ & $\mathrm{CC}$ & AA \\
\hline & 1_1052 & $\pi$ & $\mathrm{AA}$ & AA & $\pi$ \\
\hline & 1_0134 & $\mathrm{GG}$ & $\mathrm{AA}$ & $\mathrm{AA}$ & AA \\
\hline & 1__0531 & GG & $\mathrm{CC}$ & $\mathrm{CC}$ & cc \\
\hline $9.3-$ & 1_0894 & GG & cC & $\mathrm{CC}$ & $\mathrm{cc}$ \\
\hline 13.5 & 1_0757 & cc & AA & $\mathrm{AA}$ & AA \\
\hline $15.2-$ & 1_1390 & $\mathrm{AA}$ & $\mathrm{GG}$ & $\mathrm{AA}$ & $\mathrm{GG}$ \\
\hline 24.4 & 1_0563 & AA & GG & AA & GG \\
\hline $24.6 \sqrt{ }$ & 1_1388 & GG & AA & GG & AA \\
\hline 24.9 & 1_0818 & GG & AA & GG & AA \\
\hline & $11^{-} 0043$ & GG & AA & GG & AA \\
\hline & 1_0096 & AA & GG & $\mathrm{AA}$ & GG \\
\hline 25.4 & 1_0110 & GG & $\mathrm{AA}$ & GG & AA \\
\hline & 1_0550 & AA & GG & AA & GG \\
\hline & 11214 & GG & $\mathrm{CC}$ & GG & $\mathrm{cc}$ \\
\hline 26.4 & $1-0212$ & AA & $\mathrm{CC}$ & AA & cc \\
\hline 29.1 . & $1-1103$ & CC & GG & $\mathrm{CC}$ & cC \\
\hline 30.9 & 1_0955 & $\mathrm{CC}$ & AA & $\mathrm{CC}$ & $\mathrm{cc}$ \\
\hline 34.1 & 1_-0013 & AA & $\pi$ & AA & AA \\
\hline 36.0 & 11440 & $\mathrm{GG}$ & AA & GG & GG \\
\hline 36.4 & $1 \_1137$ & $\pi$ & $\mathrm{AA}$ & $\pi$ & $\pi$ \\
\hline 38.5 & 1_0322 & $\mathrm{AA}$ & $\mathrm{CC}$ & AA & AA \\
\hline 46.8 & $1-0069$ & GG & $\mathrm{AA}$ & GG & GG \\
\hline $52.3 \mathrm{~J} /$ & 10553 & CC & GG & $\mathrm{CC}$ & cc \\
\hline 59.3 & $1 \_1417$ & $\mathrm{AA}$ & $\pi$ & $\mathrm{AA}$ & $\pi$ \\
\hline
\end{tabular}

Fig. 5 Chromosomal location on linkage group VuLG11 of the cowpea consensus genetic map (Lucas et al. 2011) associated with $R k$ type resistance as revealed by SNP genotyping of two susceptible NILs (Null 1 and Null 2) and their recurrent parent CB46 (Rk resistant) and susceptible donor Chinese Red. SNP markers that are polymorphic between CB46 (grey) and Chinese Red (clear) are indicated. The full dataset of 1536 genome-wide SNP markers is provided in the Supplementary File. The genomes of the two susceptible NILs were homozygous for the CB46 background except for a common region (between the two dashed lines) that was homozygous for the susceptible donor alleles. Markers in this region (1_0134, 1_0531 and 1_0757) also flank the major QTL mapped on VuLG11 of the RIL and $\mathrm{F} 2$ populations (Figs. 3 and 4 )

incognita, but blocked female development and reproduction (Das et al. 2008). Expression profiling revealed hundreds of genes showing differential expression between the $R k$ and non- $R k$ NILs following $M$. incognita infection (Das et al. 2010). The discovery of the QTL $Q R k$-vul1.1 location in the cowpea genome region containing gene $R k$ with defined markers will enable fine mapping of $Q R k$ $v u 11.1$ and provide a framework for identifying the $R k$ trait determinant. Using the new knowledge reported here, we are currently testing candidate genes in the QTL region to determine the specific determinant of the $R k$-based nematode resistance.

Acknowledgments This work was supported by the Generation Challenge Programme of the Consultative Group on International Agricultural Research, the USAID Dry Grain Pulses CRSP (Grant EDH-A-00-07-00005), and the California Dry Bean Advisory Board.

Author Contributions statement PAR and BLH designed the research. WCM, BLH, JS, AN and JDE conducted the experiments. BLH, PAR, WCM, TJC and MRL analyzed the data. BLH and PAR wrote the manuscript and all authors reviewed the paper.

\section{Compliance with ethical standards}

Conflict of interest The authors declare that they have no conflict of interest.

Open Access This article is distributed under the terms of the Creative Commons Attribution 4.0 International License (http://creativecommons.org/licenses/by/4.0/), which permits unrestricted use, distribution, and reproduction in any medium, provided you give appropriate credit to the original author(s) and the source, provide a link to the Creative Commons license, and indicate if changes were made.

\section{References}

Abad P, Gouzy J, Aury J-M, Castagnone-Sereno P, Danchin EGJ, Deleury E, Perfus-Barbeoch L, Anthouard V, Artiguenave F, Blok VC, Caillaud M-C, Coutinho PM, Dasilva C, De Luca F, Deau F, Esquibet M, Flutre T, Goldstone JV, Hamamouch N, Hewezi T, Jaillon O, Jubin C, Leonetti P, Magliano M, Maier TR, Markov GV, McVeigh P, Pesole G, Poulain J, Robinson-Rechavi M, Sallet E, Segurens B, Steinbach D, Tytgat T, Ugarte E, van Ghelder C, Veronico P, Baum TJ, Blaxter M, Bleve-Zacheo T, Davis EL, Ewbank JJ, Favery B, Grenier E, Henrissat B, Jones JT, Laudet V, Maule AG, Quesneville H, Rosso M-N, Schiex T, Smant G, Weissenbach J, Wincker P (2008) Genome sequence of the metazoan plant-parasitic nematode Meloidogyne incognita. Nat Biotechnol 26:909-915

Atamian HS, Roberts PA, Kaloshian I (2012) High and low throughput screens with root-knot nematodes Meloidogyne spp. J Vis Exp 61:1-6

Bridge J, Page SLJ (1980) Estimation of root-knot nematode infestation levels on roots using a rating chart. Trop Pest Manag 26:296-298

Das S, DeMason DA, Ehlers JD, Close TJ, Roberts PA (2008) Histological characterization of root-knot nematode resistance in cowpea and its relation to reactive oxygen species modulation. J Exp Bot 59:1305-1313

Das S, Ehlers JD, Close TJ, Roberts PA (2010) Transcriptional profiling of root-knot nematode induced feeding sites in cowpea (Vigna unguiculata L. Walp.) using a soybean genome array. BMC Genom 11:480

Dohm JC, Lange C, Holtgräwe D, Sörensen TR, Borchardt D, Schulz B, Lehrach H, Weisshaar B, Himmelbauer H (2012) Palaeohexaploid ancestry for Caryophyllales inferred from extensive genebased physical and genetic mapping of the sugar beet genome (Beta vulgaris). Plant J 70:528-540

Ehlers JD, Hall AE (1997) Cowpea (Vigna unguiculata L. Walp.). Field Crops Res 53:187-204

Ehlers JD, Hall AE, Patel PN, Roberts PA, Matthews WC (2000a) Registrations of cultivars: registration of 'California Blackeye $27^{\prime}$ cowpea. Crop Sci 40:849-863

Ehlers JD, Matthews WC, Hall AE, Roberts PA (2000b) Inheritance of a broad-based form of root-knot nematode resistance in cowpea. Crop Sci 40:611-618

Ehlers J, Matthews W, Hall A, Roberts P (2002) Breeding and evaluation of cowpeas with high levels of broad-based resistance to root-knot nematodes. In: Fatokun C, Tarawali S, Singh B, Kormawa P, Tamo M (eds) Challenges and opportunities for enhancing sustainable cowpea production Proceedings of the World Cowpea Conference. The International Institute of Tropical Agriculture (IITA), Ibadan, pp 41-51 
Ehlers JD, Sanden BL, Frate CA, Hall AE, Roberts PA (2009) Registration of 'California Blackeye 50' cowpea. J Plant Regist 3:236-240

Fery RL, Dukes PD (1980) Inheritance of root-knot nematode resistance in cowpea (Vigna unguiculata [L.] Walp.). J Am Soc Hortic Sci 105:671-674

Fery RL, Dukes PD, Thies JA (1994) Characterization of new sources of resistance in cowpea to the southern root-knot nematode. HortScience 29:678-679

Hall AE, Frate CA (1996) Blackeye bean production in California. Publication 21518. Division of Agriculture and Natural Resources, University of California

Hall AE, Cisse N, Thiaw S, Elawad HOA, Ehlers JD, Ismail AM, Fery RL, Roberts PA, Kitch LW, Murdock LL, Boukar O, Phillips RD, McWatters KH (2003) Development of cowpea cultivars and germplasm by the Bean/Cowpea CRSP. Field Crops Res 82:103-134

Helms D, Panella L, Buddenhagen IW, Tucker CL, Gepts PL (1991) Registration of 'California Blackeye 46' cowpea. Crop Sci 31:1703

Hoagland DR, Arnon DI (1950) The water-culture method for growing plants without soil. California Agricultural Experiment Station Circular 347. University of California, Berkely, CA, USA

Huynh B-L, Ehlers JD, Ndeve A, Wanamaker S, Lucas MR, Close TJ, Roberts PA (2015) Genetic mapping and legume synteny of aphid resistance in African cowpea (Vigna unguiculata L. Walp.) grown in California. Mol Breed 35:1-9

Li H, Ye G, Wang J (2007) A modified algorithm for the improvement of composite interval mapping. Genetics 175:361-374

Lucas MR, Diop N-N, Wanamaker S, Ehlers JD, Roberts PA, Close TJ (2011) Cowpea-soybean synteny clarified through an improved genetic map. Plant Genome 4:218-225

Lucas MR, Ehlers JD, Roberts PA, Close TJ (2012) Markers for quantitative inheritance of resistance to foliar thrips in cowpea. Crop Sci 52:2075-2081

Muchero W, Diop NN, Bhat PR, Fenton RD, Wanamaker S, Pottorff M, Hearne S, Cisse N, Fatokun C, Ehlers JD, Roberts PA, Close TJ (2009) A consensus genetic map of cowpea [Vigna unguiculata (L) Walp.] and synteny based on EST-derived SNPs. Proc Natl Acad Sci USA 106:18159-18164

Muchero W, Ehlers JD, Close TJ, Roberts PA (2011) Genic SNP markers and legume synteny reveal candidate genes underlying QTL for Macrophomina phaseolina resistance and maturity in cowpea Vigna unguiculata (L) Walp. BMC Genom 12:8

Olowe T (2004) Occurrence and distribution of root-knot nematodes, Meloidogyne spp., in cowpea growing areas of Nigeria. Nematology 6:811-817

Omwega CO, Thomason IJ, Roberts PA (1988) A nondestructive technique for screening bean germ plasm for resistance to Meloidogyne incognita. Plant Dis 72:970-972

Ouédraogo JT, Ouédraogo M, Gowda BS, Timko MP (2012) Development of sequence characterized amplified region (SCAR) markers linked to race-specific resistance to Striga gesnerioides in cowpea (Vigna unguiculata L.). Afr J Biotechnol 11:12555-12562

Ouédraogo JT, Gowda BS, Jean M, Close TJ, Ehlers JD, Hall AE, Gillaspie AG, Roberts PA, Ismail AM, Bruening G, Gepts P, Timko MP, Belzile FJ (2002) An improved genetic linkage map for cowpea (Vigna unguiculata L.): combining AFLP, RFLP, RAPD, biochemical markers, and biological resistance traits. Genome 45:175-188

Payne RW, Harding SA, Murray DA, Soutar DM, Baird DB, Glaser AI, Channing IC, Welham SJ, Gilmour AR, Thompson R, Webster R (2008) GENSTAT release 11 reference manual. Parts 1, 2 and 3. VSN International: Hemel Hempstead, UK

Petrillo MD, Roberts PA (2005) Isofemale line analysis of Meloidogyne incognita virulence to cowpea resistance gene $R k$. J Nematol 37:448-456

Petrillo MD, Matthews WC, Roberts PA (2006) Dynamics of Meloidogyne incognita virulence to resistance genes $R k$ and $R k 2$ in cowpea. J Nematol 38:90-96

Pottorff M, Wanamaker S, Ma YQ, Ehlers JD, Roberts PA, Close TJ (2012) Genetic and physical mapping of candidate genes for resistance to Fusarium oxysporum f. sp. tracheiphilum race 3 in cowpea [Vigna unguiculata (L.) Walp]. PLoS One 7:e41600

Pottorff MO, Li G, Ehlers JD, Close TJ, Roberts PA (2014) Genetic mapping, synteny, and physical location of two loci for Fusarium oxysporum f. sp. tracheiphilum race 4 resistance in cowpea [Vigna unguiculata (L.) Walp]. Mol Breed 33:779-791

Roberts PA, Frate CA, Matthews WC, Osterli PP (1995) Interactions of virulent Meloidogyne incognita and Fusarium wilt on resistant cowpea genotypes. Phytopathology 85:1288-1295

Roberts PA, Matthews WC, Ehlers JD (1996) New resistance to virulent root-knot nematodes linked to the $R k$ locus of cowpea. Crop Sci 36:889-894

Roberts PA, Matthews WC, Ehlers JD (2005) Root-knot nematode resistant cowpea cover crops in tomato production systems. Agron J 97:1626-1635

Sawadogo A, Thio B, Kiemde S, Drabo I, Dabire C, Ouedraogo J, Mullens TR, Ehlers JD, Roberts PA (2009) Distribution and prevalence of parasitic nematodes of cowpea (Vigna unguiculata) in Burkina Faso. J Nematol 41:120-127

Tamulonis JP, Luzzi BM, Hussey RS, Parrot WA, Boerma HR (1997) DNA markers associated with resistance to javanese root-knot nematode in soybean. Crop Sci 37:783-788

Thomason IJ, McKinney HE (1960) Reaction of cowpeas, Vigna sinensis to root-knot nematodes, Meloidogyne spp. Plant Dis Report 44:51-53

Van Os H, Stam P, Visser RGF, Van Eck HJ (2005) RECORD: a novel method for ordering loci on a genetic linkage map. Theor Appl Genet 112:30-40

Wang J (2009) Inclusive composite interval mapping of quantitative trait genes. Acta Agron Sinica 35:239-245

Xu X, Zeng L, Tao Y, Vuong T, Wan J, Boerma R, Noe J, Li Z, Finnerty S, Pathan SM, Shannon JG, Nguyen HT (2013) Pinpointing genes underlying the quantitative trait loci for root-knot nematode resistance in palaeopolyploid soybean by whole genome resequencing. Proc Natl Acad Sci USA 110:13469-13474

Yang J, Zhu J, Williams RW (2007) Mapping the genetic architecture of complex traits in experimental populations. Bioinformatics 23:1527-1536

Zhang D, Wu J, Zhang Y, Shi C (2012) Phenotypic and candidate gene analysis of a new floury endosperm mutant (osagpl2-3) in rice. Plant Mol Biol Rep 30:1303-1312 